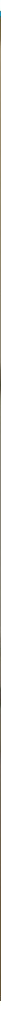

\title{
Wissensgenerierende onkologische Versorgung schaffen
}

\author{
Interview mit Prof. Dr. Olaf Ortmann, Direktor der Klinik \\ für Frauenheilkunde und Geburtshilfe, Universität Regensburg, \\ Caritas-Krankenhaus St. Josef
}

Die Stakeholder der Onkologie treiben trotz COVID-Krise die Umsetzung der Nationalen Dekade gegen Krebs voran. Prof. Dr. Olaf Ortmann sieht in der wissensgenerierenden onkologischen Versorgung einen wichtigen Baustein für eine flächendeckende hochwertige Krebsmedizin. Dafür setzt er sich unter anderem in der Arbeitsgruppe „Wissen generieren durch Vernetzung von Forschung und Versorgung" ein.

best practice onkologie: Sie sind Direktor der Klinik für Frauenheilkunde und Geburtshilfe der Universität Regensburg, wie sind Sie zu dieser Fachrichtung gelangt, und was fasziniert Sie daran?

O. Ortmann: Mich hat bereits im Laufe meines Studiums die Frauenheilkunde interessiert. Ich habe meine Doktorarbeit in der Klinik für Frauenheilkunde und Geburtshilfe der Universität Lübeck gemacht und habe mich dort mit hormo- neller Regulation von verschiedenen Regelkreisen beschäftigt. Das hat mich vom wissenschaftlichen Ansatz her zur Frauenheilkunde gebracht. In der Klinik habe ich noch während meines Studiums erfahren, wie vielfältig die Frauenheilkunde ist. Insbesondere ist interessant, dass man sowohl operative als auch konservative Tätigkeiten miteinander verbinden kann und das hat mich dann auch im Laufe meiner beruflichen Tätigkeit zu dem Schwerpunkt der Krebsmedizin gebracht. Es fasziniert mich eigentlich bis heute, dass wir in der gynäkologischen Onkologie mehr einen ganzheitlichen Ansatz haben als vielleicht in anderen Fächern.

best practice onkologie: Neben Ihrer Klinik leiten Sie noch das Onkologische Zentrum Regensburg und engagieren sich in vielen medizinischen Fachgesellschaften. Wie bekommen Sie all diese Verantwortlichkeiten unter einen Hut?
O. Ortmann: Man wächst natürlich mit der Zeit in die Aufgaben hinein. Diese alle unter einen Hut zu bekommen, schaffe ich natürlich nicht ganz allein, sondern ich habe Teams aufgebaut, die mir hier im Klinikum der Universität Regensburg und dem Caritas-Krankenhaus St. Josef Kontakte zu vielen Mitarbeitern ermöglichen, die das alles mittragen und gestalten. Als Teamleader bin ich letztendlich auch nur ein Teil des Teams, wobei ich hier und da häufig tonangebend sein muss. Es hängt alles davon $a b$, wie gut man so ein Team formiert und sich mit ihm zusammen weiterentwickelt.

best practice onkologie: Während der Hochphase der COVID-19-Krise mussten besonders die Krebspatienten zurückstecken - wie haben Sie diese Zeit erlebt?

O. Ortmann: Das ist in der Tat eine besondere Belastungssituation gewesen. Es sind nicht nur Krebspatienten, die unter 
der COVID-Krise „leiden“ mussten, sondern auch andere Patienten mit elektiven und akuten Erkrankungen, wie zum Beispiel Patienten mit Herzerkrankungen oder neurovaskulären Problemen. Das lag nicht nur an den Einschränkungen, die uns von behördlicher Seite oder von Ministerien auferlegt wurden. Es wurde beobachtet, dass die Patienten tatsächlich seltener die klinische Versorgung aufgesucht haben, weil sie Angst hatten, sich anzustecken. Ich persönlich habe es im Bereich der Krebsmedizin - sowohl im Krebszentrum als auch in meiner eigenen Klinik - so gehalten, dass Krebspatienten nach wie vor uneingeschränkt weiter behandelt wurden, bis auf die Berücksichtigung besonderer Sicherheitskriterien in der COVID-Phase.

\section{》) Es wurde beobachtet, dass Patienten seltener die klinische Versorgung aufgesucht haben, weil sie Angst hatten, sich anzu- stecken}

best practice onkologie: Ist der Alltag jetzt wieder eingekehrt?

O. Ortmann: Der Alltag ist noch nicht wieder eingekehrt, muss man sagen. Wir haben nach wie vor Begrenzungen und Beschränkungen, die von behördlichen Auflagen abhängen. Das bedeutet, wir müssen bestimmte Kapazitäten immer noch für COVID-Patienten freihalten, und das kann die Kapazitäten für Krebspatienten schon einschränken.

best practice onkologie: Inwiefern hat die COVID-Krise Einfluss auf die nationale Dekade gegen Krebs? Hat sie den Zeitplan verzögert?

O. Ortmann: Ja, die COVID-Krise hat sicherlich zu einer gewissen Verzögerung geführt, weil Anwesenheitssitzungen nicht in dem Maße eingehalten werden konnten, wie das vorgesehen war. Das ist, denke ich, nachvollziehbar. Wir haben, wo es möglich war, auf Videokonferenzen umgeschaltet, sodass eigentlich die Fortführung der Arbeit in vielen der Unterarbeitsgruppen trotzdem stattgefunden hat. Insgesamt haben wir nur eine relativ leichte Verzögerung in Kauf nehmen müssen.

best practice onkologie: Hat die COVID-Krise neue Ideen für Kooperationen gebracht?

O. Ortmann: Es ist sozusagen ein Sekundäreffekt der COVID-Krise entstanden: Wir haben auf Initiative von Herrn Prof. Michael Baumann, Direktor des Deutschen Krebsforschungszentrums und Moderator der Nationalen Dekade gegen Krebs, gemeinsam mit der Deutschen Krebshilfe und der Deutschen Krebsgesellschaft eine Task Force "Corona und Krebs“ gegründet. Darin haben wir während der COVID-Phase mit verschiedenen Kooperationspartnern geprüft, was die Auswirkungen der Pandemie auf die Versorgung von Krebserkrankten sind. Um ein flächendeckendes Bild zu erfahren, wo Einschränkungen durch die COVID-Pandemie stattgefunden haben, hat dies in den großen Comprehensive Cancer Centers und mit einer gewissen Latenz auch in den großen von der Deutschen Krebsgesellschaft zertifizierten onkologischen Zentren stattgefunden. Wir haben solche Einschränkungen tatsächlich festgestellt, die sehr deutlich waren im Bereich der Früherkennung - zum Beispiel war das Mammographiescreening phasenweise völlig eingestellt - aber auch im Bereich der Tumornachsorge. Weniger sind die Einschränkungen eigentlich bei der akuten Versorgung von Krebserkrankten aufgetreten. Hier konnte die Ver- sorgung durchweg relativ gut gewährleistet werden, sodass im klinischen Bereich oder auch in Schwerpunktpraxen die Einschränkungen eher punktuell auftraten.

\section{》) Wir haben Einschrän- kungen festgestellt, die sehr deutlich waren im Bereich der Früher- kennung, aber auch im Bereich der Tumornach- sorge}

best practice onkologie: Die Nationale Dekade gegen Krebs hat Ende 2019 begonnen - welche Mission steckt dahinter?

O. Ortmann: Die Nationale Dekade wurde vom Bundesministerium für Bildung und Forschung (BMBF) in Zusammenarbeit mit dem Bundesministerium für $\mathrm{Ge}$ sundheit (BMG) und vielen weiteren Partnern initiiert. Wir haben eine gemeinsame Erklärung formuliert, die unsere Mission, zentrale Ziele und Handlungsfelder beinhaltet. Für mich ist hier besonders hervorzuheben, dass wir die Ergebnisse der Forschung rasch zu den Menschen bringen und die Erfahrung und Erkenntnisse aus der onkologischen Versorgung der Krebskranken für die Forschung nutzen wollen, um damit letztendlich die Versorgung

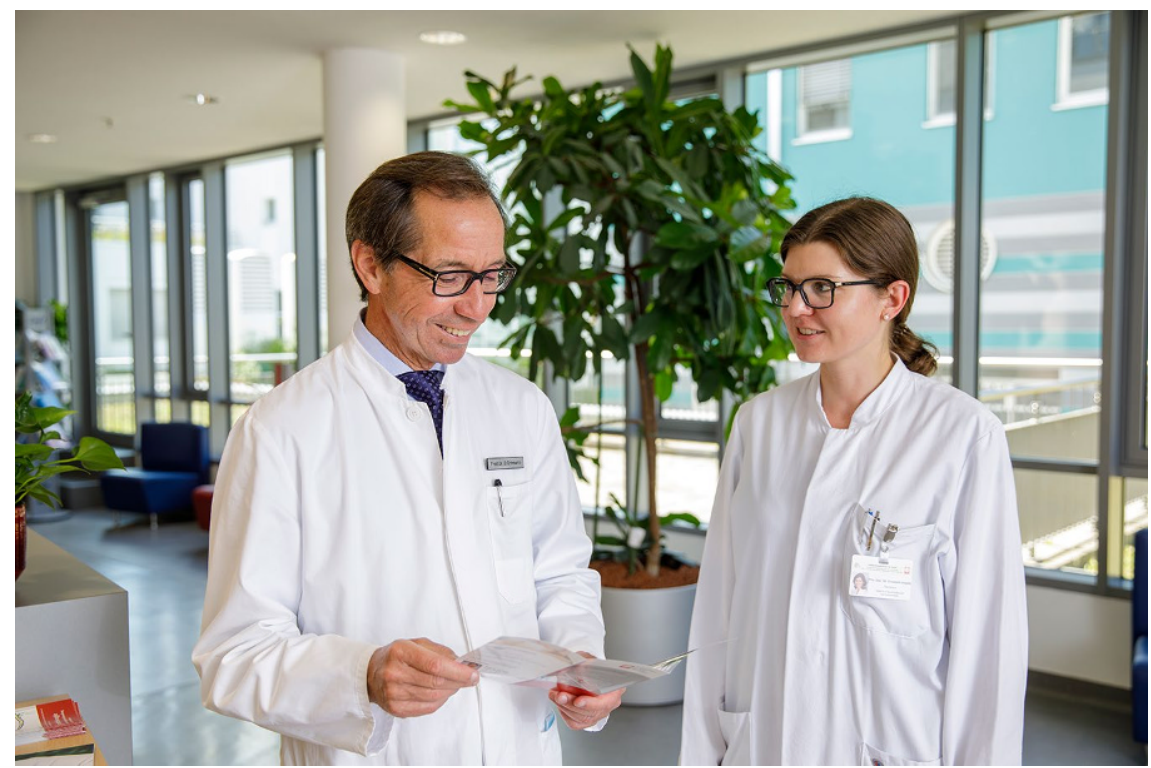

Ein herzliches Betriebsklima ist auch in der Klinik von unschätzbarem Wert 


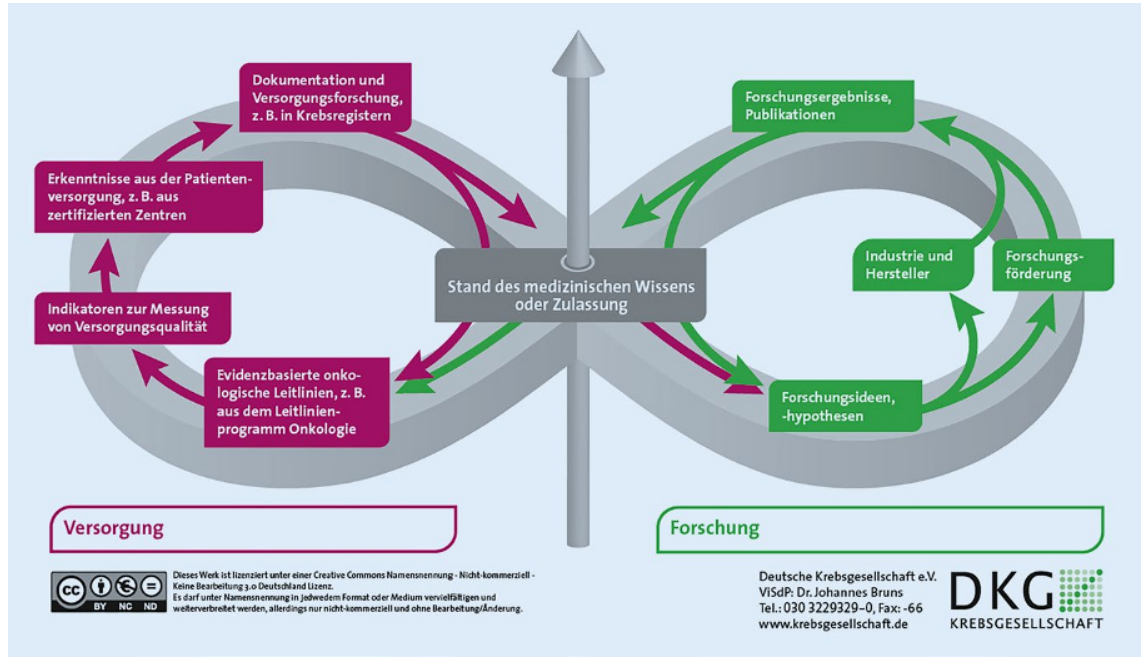

„Wissensgenerierende onkologische Versorgung. (Quelle: Deutsche Krebsgesellschaft) [1]"

weiter zu verbessern. Wir wollen, dass es den Krebspatienten besser geht, dass sie die Behandlung akzeptieren und gut durchführen können, was somit auch zu besseren Behandlungsergebnissen führt. Die Patienten stehen im Mittelpunkt, weshalb Patientenvertreter auch Mitglieder im Strategiekreis der Nationalen Dekade gegen Krebs sind. Ich halte es für besonders wichtig, deren Perspektive miteinzubeziehen. $\mathrm{Zu}$ den wichtigen Zielen gehören auch die Forschung und die schnelle Umsetzung der Ergebnisse in die flächendeckende onkologische Versorgung sowie die Krebsprävention, weil wir wissen, dass durch Prävention ein Großteil der Krebserkrankungen vermieden werden kann. Wir wollen, dass alle Menschen in Deutschland Zugang zu einer qualitativ hochwertigen onkologischen Versorgung erhalten. Wir wollen das Bewusstsein für Krebsprävention in der Bevölkerung verbessern, und Deutschland soll ein internationaler führender Standort in der patientenorientierten Krebsforschung und der onkologischen Versorgung sein.

best practice onkologie: Konnten schon erste Schritte umgesetzt werden?

O. Ortmann: Ja, wir haben erste Schritte umgesetzt. Initial wurden Treffen des so genannten Strategiekreises einberufen, in den wichtige Stakeholder in der Onkologie berufen worden sind. Wir haben die Arbeit in der Weise strukturiert, dass wir drei große Arbeitsgruppen gegründet haben: die Arbeitsgruppe „Prävention“, die Arbeitsgruppe "Große ungelöste Fragen der Krebsforschung" und ten Themen gewidmet, die sie in den nächsten Jahren prädominant bearbeiten wollen, die Arbeitsgruppe "Große ungelöste Fragen der Krebsforschung" u. a. den Themen Tumorheterogenität, klonale Evolution und Therapieresistenz.

best practice onkologie: Welche Ziele hat sich Ihre Arbeitsgruppe gesetzt?

O. Ortmann: Ein Ziel meiner Arbeitsgruppe "Wissen generieren durch Vernetzung von Forschung und Versorgung " ist es, die Ergebnisse aus der Spitzenforschung schneller zum Patienten zu bringen, das heißt also, diesen Translationsprozess zu verbessern. Außerdem wollen wir analysieren, ob der Effekt, den wir in klinischen Studien gesehen haben, auch tatsächlich in der Versorgung bei den Patienten ankommt. Das zweite Ziel ist es, zu erarbeiten, was wir aus den Daten, die wir in der Versorgung von Krebspatienten sehen, lernen können und welche Rückschlüsse wir für die Verbesserung der Versorgung ziehen können. Ein international großes Thema ist in diesem Zusammenhang die Forschung mit Real-World-Data. Aber deren Wert wird häufig überschätzt. Wir wollen dagegen sehr strukturiert Daten erheben, was man zum Beispiel mit dem Instrument der klinischen Krebsregistrierung oder anderen Datenerhebungsinstrumenten unter anderem in zertifizierten Krebszentren der Deut-

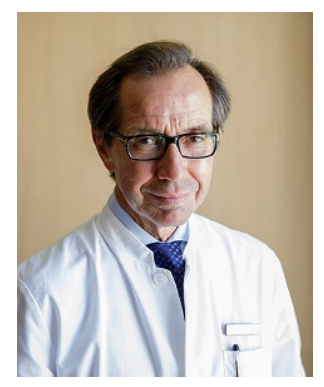

\section{Zur Person}

Prof. Dr. Olaf Ortmann ist Direktor der Klinik für Frauenheilkunde und Geburtshilfe, Universität Regensburg, Caritas-Krankenhaus St. Josef. Er leitet das Onkologische Zentrum Regensburg und ist unter anderem Vorstandsmitglied des Comprehensive Cancer Centers Ostbayern. Der gebürtige Lübecker studierte in seiner Heimat Medizin und gelangte nach Stationen u. a. in den USA und Marburg an die Universität Regensburg, wo er seit 2003 den Lehrstuhl für Frauenheilkunde und Geburtshilfe innehat. Seine wissenschaftlichen Schwerpunkte liegen im Bereich der zielgerichteten Therapie und der registerbasierten Forschung bei gynäkologischen Krebserkrankungen. Er setzt sich auch in der Deutschen Krebsgesellschaft (DKG) für eine bessere Versorgung der Krebspatienten ein, zunächst als Sprecher der wissenschaftlichen Arbeitsgemeinschaften, als DKG-Vizepräsident und von 2018-2020 als Präsident der DKG. Heute ist er weiterhin DKG-Vorstandsmitglied. Er ist Mitglied im Strategiekreis der Nationalen Dekade gegen Krebs und leitet gemeinsam mit der parlamentarischen Staatssekretärin Sabine Weiß die Arbeitsgruppe „Wissen generieren durch Vernetzung von Forschung und Versorgung". Darüber hinaus ist er Mitglied in vielen medizinischen Fachgesellschaften und unterstützt als Herausgeber und Mitherausgeber mehrere Fachzeitschriften. 
schen Krebsgesellschaft erreichen kann. Aus diesen Daten hoffen wir Erkenntnisse zu gewinnen, die dann sinnvoll in veränderte Versorgungskonzepte umgesetzt werden können. Dazu gehört auch, Datenbanken und Datenstrukturen, die in Deutschland geschaffen wurden, genau $\mathrm{zu}$ analysieren und $\mathrm{zu}$ schauen, wie man diese am besten zusammenführt, um ein Instrument für wissensgenerierende Versorgung zu schaffen. Das bedeutet, wir müssen die Heterogenität von Registern, die derzeit herrscht, besser strukturieren, um einen sinnvollen Erkenntnisgewinn zu ermöglichen.

\section{》) Wir müssen die Hetero- genität von Registern, die derzeit herrscht, besser strukturieren, um einen sinnvollen Erkenntnisge- winn zu ermöglichen}

best practice onkologie: Sie setzen sich besonders für die Versorgungsforschung bei gynäkologischen Krebserkrankungen ein. Was wurde hier schon erreicht, und welche Herausforderungen gilt es noch zu meistern?

O. Ortmann: Wir haben insbesondere am populationsbasierten klinischen Krebsregister im Einzugsgebiet des Tumorzentrums in Regensburg gearbeitet. Damit können wir einerseits Versorgungsforschung im klassischen Sinne betreiben. Diese hat den Anspruch, Versorgungsqualität $\mathrm{zu}$ überprüfen und bestimmte Versorgungsdefizite in Regionen oder bei bestimmten Tumorentitäten aufzuzeigen und daraufhin die Versorgung zu verbessern. Das ist es aber nicht, was wir hier ausschließlich beabsichtigen. Wir wollen darüber hinaus durch registerbasierte Forschung mit klinischen Krebsregistern Wissen generieren zu Fragen, für die Evidenz aus klinischen Studien fehlt. Wenn es zum Beispiel Unklarheiten zu operativen Strategien bei bestimmten Tumorstadien von Gebärmutterhalskrebs oder von Gebärmutterschleimhautkrebs gibt, weil wir keine ausreichende Anzahl von prospektiven randomisierten Studien haben, dann schauen wir uns qualitativ hochwertige Daten aus klinischen Krebsre-

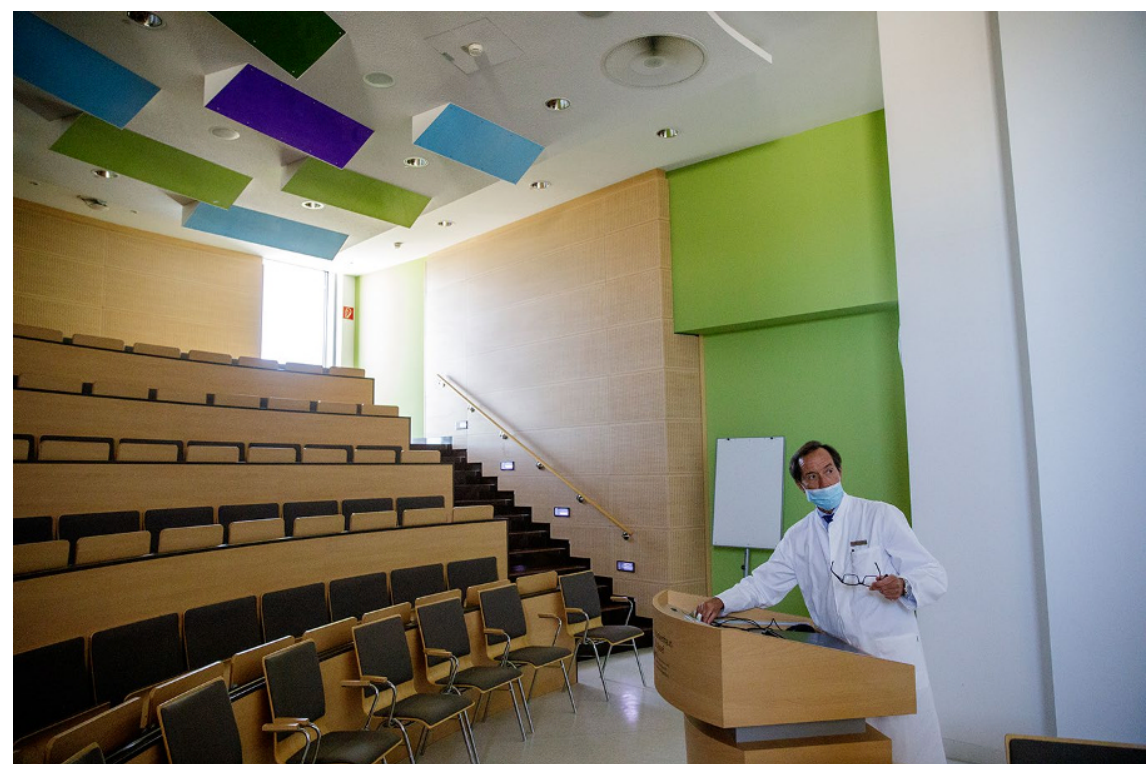

Vorträge und Vorlesungen sind ein wichtiger Teil der medizinisch-onkologischen Aus- und Weiterbildung

gistern an. Diese analysieren wir und leiten daraus dann bestimmte Erkenntnisse ab. Derzeit führen wir das zum Beispiel in Studien zu Brustkrebserkrankungen mit Daten aus zertifizierten Brustkrebszentren durch. In dem Projekt nutzen wir bestimmte Datenbanken der Deutschen Krebsgesellschaft. Wir arbeiten dabei mit verschiedenen Zentren zusammen und erarbeiten gemeinsam Fragen, die sonst mit anderen Studienkonzepten bisher nicht geklärt werden konnten, und beantworten sie mit den Mitteln einer hochwertigen registerbasierten Forschung.

\section{》) Durch registerbasierte Forschung Wissen gene- rieren zu Fragen, für die Evidenz aus klinischen Studien fehlt}

best practice onkologie: Es entstehen immer mehr Spitzenzentren der Krebsmedizin in Deutschland. Wie werden niedergelassene Ärzte und Praxen sinnvoll mit in die Versorgung eingebunden, vielleicht am Beispiel des University Cancer Centers Regensburg als Teil des Comprehensive Cancer Centers Ostbayern?

O. Ortmann: Als Zentrum wird häufig verstanden, dass das eine Institution an ei- nem Ort ist, wo dann alles getan wird. Das entspricht aber überhaupt nicht dem Bild der gesamten Versorgung von Krebspatienten. Das deutsche Gesundheitssystem ist relativ komplex. Alle Komponenten eines Krebszentrums befinden sich nicht in einem Gebäude. Es sollte immer ein Netzwerk sein. Ein Zentrum ist also nicht ein Standort, sondern Zentrum bedeutet das Zusammenbringen von verschiedenen Leistungserbringern in der Krebsmedizin, die alle an der Versorgung von Krebserkrankten beteiligt sind. Und das umfasst auch die Einbindung von niedergelassenen Kollegen, die als Kooperationspartner in diesem Zentrum mit agieren. Ganz konkret führen wir Tumorkonferenzen, auch virtuell, in Anwesenheit von niedergelassenen Kollegen durch. Sie können ihre Vorschläge und Kommentare einbringen, die dann auch bei der Therapieentscheidung berücksichtigt werden, die im Zentrum gefällt wird.

best practice onkologie: Ziel der nationalen Dekade gegen Krebs ist unter anderem die wohnortnahe onkologische Versorgung nach modernsten Standards, wie nah sind wir in Deutschland diesem Ziel schon gekommen?

O. Ortmann: Diesem Ziel sind wir, glaube ich, schon sehr nah gekommen. Die Nationale Dekade gegen Krebs ist aus meiner Sicht ein wirklicher Schmelztiegel geworden für alle, die an Krebsmedizin im weitesten Sinne beteiligt sind. Das ist 


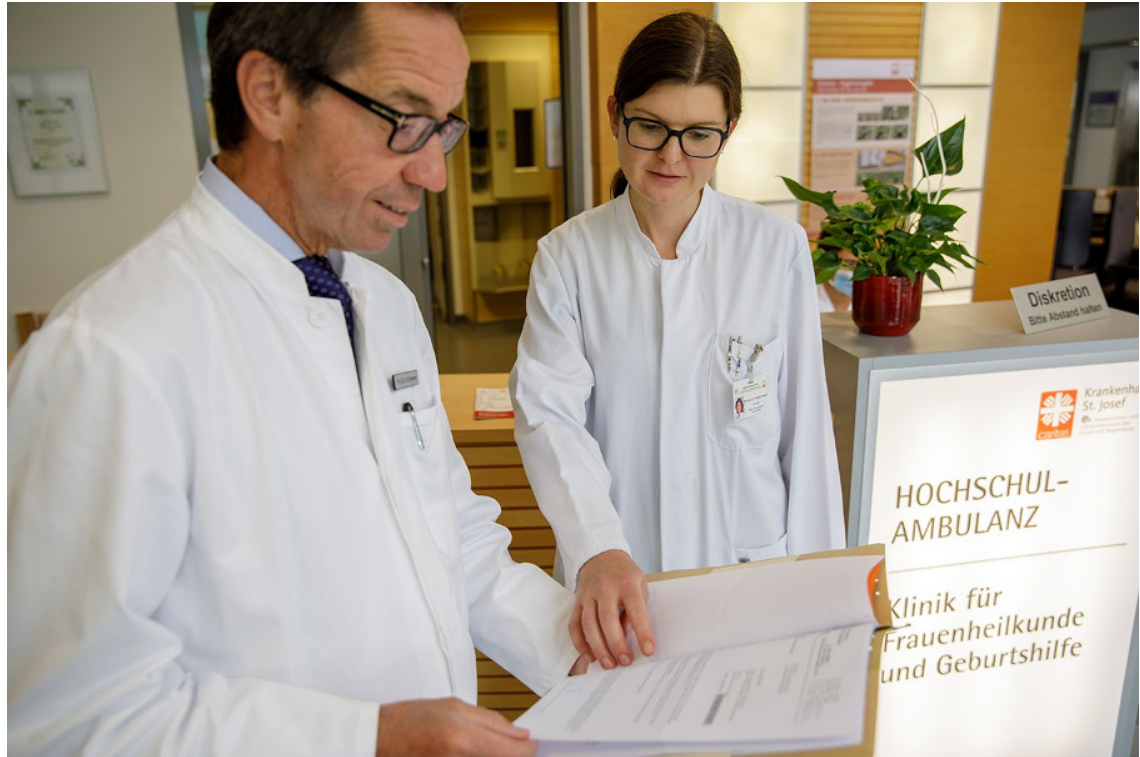

In der Hochschulambulanz wird jede Gelegenheit genutzt, eine Frage mit dem Chef zu klären

eine hervorragende Initiative, um die ganze Komplexität zu betrachten. Und nur wenn man so vorgeht, wird man letztendlich die Versorgung und die Krebsmedizin voranbringen. Einzelne Institutionen werden das nie schaffen, sie können vielleicht für eine begrenzte Anzahl von Krebspatienten oder in der Krebsforschung sehr hochwertige Leistung erbringen, aber wir haben vor, exzellente Krebsmedizin allen Bürgern in Deutschland, die an Krebs erkrankt sind oder Risiken für Krebs haben, zugänglich zu machen. Und da sind wir - natürlich nicht nur aufgrund der Dekade, sondern auch mit dem, was sich in Deutschland in den letzten beiden Jahrzehnten entwickelt hat - schon ein großes Stück vorangekommen. Sicherlich hat dazu entscheidend die Zentrumsentwicklung beigetragen, die wir im Rahmen des Zertifizierungssystems der Deutschen Krebsgesellschaft auf den Weg gebracht haben und die auch im Nationalen Krebsplan Bestandteil in einem der wichtigen Handlungsfelder geworden ist. In diesen Zentren werden derzeit bereits ungefähr $50 \%$ aller inzidenten Krebserkrankungen behandelt, mit langsam zunehmender Tendenz - das ist ein Erfolg!

Das zweite erfolgreiche Modell sind die onkologischen Spitzenzentren, die so genannten Comprehensive Cancer Centers. Diese werden von der Deutschen Krebshilfe und der Deutschen Krebsgesellschaft zertifiziert - und dieser Schulterschluss hat eigentlich dazu geführt, dass diese
Netzwerke erst richtig effizient werden können. Das heißt, diese onkologischen Spitzenzentren betreiben ihre Netzwerke, in diese Netzwerke sind dann andere, zum Beispiel nichtakademische onkologische Zentren eingebunden. Diese wiederum ermöglichen mit ihren Netzwerken zwischen kleineren Krankenhäusern und niedergelassenen Ärzten dann auch $\mathrm{Pa}$ tienten weitgehend flächendeckend $\mathrm{Zu}$ gang zu onkologischer Spitzenmedizin.

\section{I) Die nationale Dekade gegen Krebs ist ein wirk- licher Schmelztiegel geworden für alle, die an Krebsmedizin im weites- ten Sinne beteiligt sind}

best practice onkologie: Krebs ist nicht nur ein nationales Problem. Wie wird die europaweite Zusammenarbeit vorangetrieben?

O. Ortmann: Wir haben einige ganz konkrete Initiativen: Wir veranstalten vonseiten der Deutschen Krebsgesellschaft mit der Union for International Cancer Control (UICC) seit vielen Jahren so genannte European Round Table Meetings. Dazu laden wir jedes Jahr europäische Nationen ein, um uns mit ihnen zu bestimmten
Themen auszutauschen und gemeinsame Strategien festzulegen. Dadurch ist die Zusammenarbeit mit der UICC geschärft worden, und wir sind in engem Kontakt mit den European Cancer Leagues (ECL). Außerdem sind einige europäische Initiativen auf dem Weg. So hat die EU-Ratspräsidentschaft Deutschlands Krebs zum Thema gemacht, und es kann ein europäischer Krebsplan neu aufgelegt und formuliert werden. Natürlich gibt es weiterhin auch die großen europäischen Fachgesellschaften in den verschiedenen Fachbereichen der Krebsmedizin. Das Engagement in Europa bezieht sich jetzt auch sehr stark auf das, was wir in der nationalen Dekade gegen Krebs thematisieren: auf das Vorantreiben der Krebsmedizin inklusive der Versorgungsstrukturen. Und da gibt es in Europa sehr unterschiedliche Verhältnisse, und da können insbesondere Nationen, die größere ökonomische oder strukturell bedingte Schwierigkeiten haben, von ihren Nachbarn lernen.

best practice onkologie: Herr Professor Ortmann, vielen Dank für das interessante Gespräch.

Das Interview führte: Sabrina Kempe, Dresden

Fotos: () Max Kratzer

Literatur

1. Ortmann O et al (2019) Wissen generieren durch Vernetzung von Forschung und Versorgung. Forum 34(6):516-552 


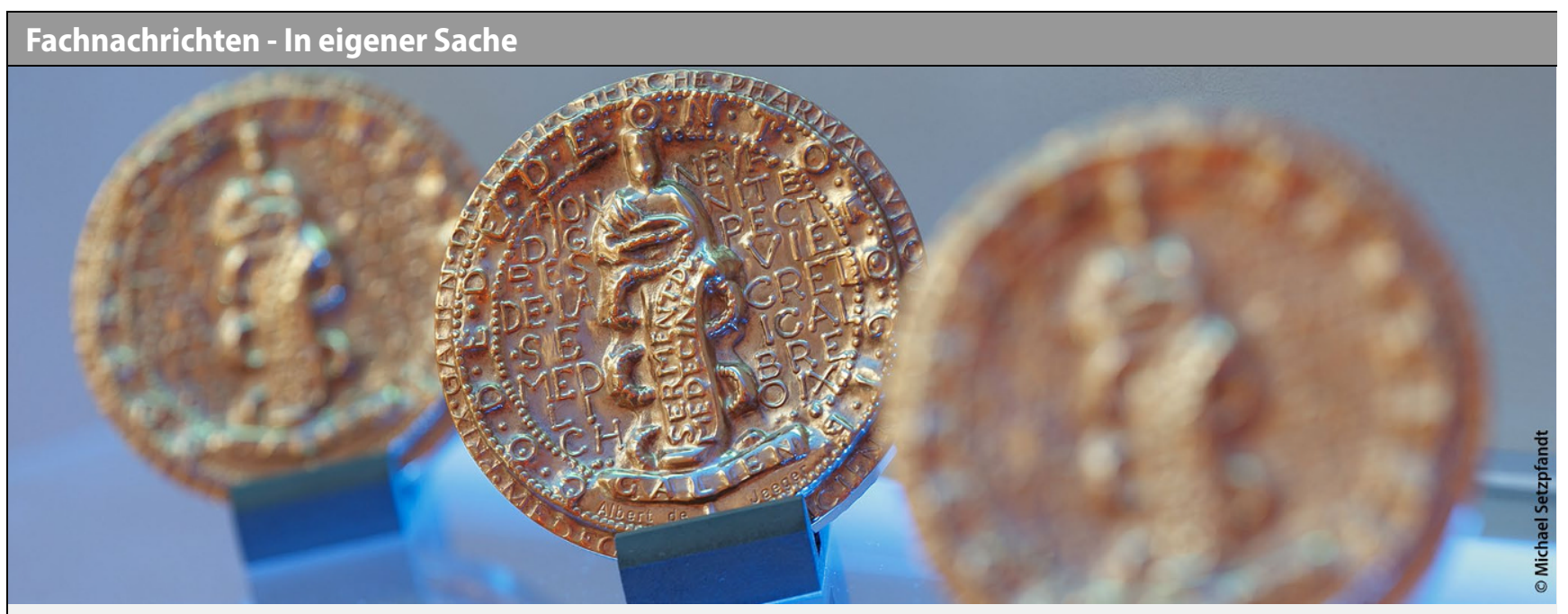

\section{Galenus-von-Pergamon-Preis 2020: 14 Arzneimittel sind im Rennen}

\section{Mit dem von der Springer Medizin Verlag GmbH gestifteten Galenus-von-Pergamon-Preis werden seit 1985 in Deutschland herausragende Arzneimittelinnovationen gewürdigt. \\ Der Preis wird in den Kategorien „Primary Care“, „Specialist Care“ und „Orphan Drugs“ vergeben. Über die Zuerkennung entscheidet eine unabhängige Expertenjury. Überreicht wird der Preis bei einem Festakt am 22. Oktober in Berlin. Hier stellen wir Ihnen zwei Kandidaten vor:}

\section{Erleada $^{\circledR}$}

\section{(Apalutamid)}

Für Patienten mit metastasiertem hormonsensitivem Prostatakarzinom ( $\mathrm{mHSPC}$ ) besteht trotz zwei zugelassener Therapieoptionen ein hoher medizinischer Bedarf an Behandlungsalternativen. Die im Januar 2020 erteilte Indikationserweiterung für den oralen Androgenrezeptor (AR)Inhibitor Apalutamid (Erleada ${ }^{\circledR}$ ) von Janssen ist daher ein wichtiger Schritt in der Therapie. Apalutamid ermöglicht in Kombination mit einer Androgendeprivationstherapie (ADT) einem breiten Spektrum von $\mathrm{mHSPC}$-Patienten eine effektive antihormonelle und dabei gut verträgliche Behandlung, wie die Zulassungsstudie TITAN gezeigt hat. Die Kombination Apalutamid/ADT kann, unter Berücksichtigung von Warnhinweisen und Kontraindikationen, grundsätzlich bei allen mHSPC-Patienten, unabhängig z. B. von lokalen Vortherapien oder dem Progressionsrisiko, angewandt werden. Das erleichtert Ärzten die Therapie.

In der TITAN-Studie erhielten die Patienten entweder Apalutamid (240mg/Tag) plus ADT oder Placebo/ADT. Patienten im Apalutamid/ADTArm hatten ein um $33 \%$ geringeres Sterberisiko als jene im Placebo/ADT$\operatorname{Arm}(p=0,005)$. Das mediane Gesamtüberleben war zum Zeitpunkt der zulassungsrelevanten Interimsanalyse in beiden Studienarmen noch nicht erreicht. Die 2-Jahres-Überlebensrate betrug nach einer medianen Beobachtungszeit von 22,7 Monaten 82,4\% im Apalutamid/ADT-Arm und $73,5 \%$ im Placebo/ ADT-Arm.

\begin{abstract}
Vitrakvi $^{\otimes}$ (Larotrectinib)

Die Identifizierung definierter Treibermutationen erlaubt es immer häufiger, mit spezifischen Wirkstoffen gezielt am ursächlichen pathogenetischen Mechanismus der Tumorentwicklung einzugreifen. Ein starker onkogener Treiber entsteht bei Fusion des NTRK (neurotrophe TyrosinRezeptor-Kinase)-Gens mit weiteren Genen. Diese NTRK-Genfusionen kodieren für dauerhaft aktivierte Fusionsproteine, die unreguliertes Zellwachstum induzieren. Mit Larotrectinib (Vitrakvi ${ }^{\circledR}$ ) von Bayer Vital ist seit September 2019 ein spezifischer Inhibitor der TRK (TropomyosinRezeptor-Kinasen) A, B und C erhältlich, der die Überaktivierung potenziell onkogener Signalwege blockiert. Larotrectinib ist die erste in der EU zugelassene Substanz, die unabhängig von der Art eines soliden Tumors indiziert ist. Anwendungsvoraussetzung ist der Nachweis einer NTRK-Genfusion im Tumor.

Die Behandlung mit Larotrectinib führte in drei Studien der Phase I und II bei intensiv vorbehandelten erwachsenen und pädiatrischen Patienten mit über 20 unterschiedlichen Tumoren zu einer Gesamtansprechrate von 79\% und einer langen Ansprechdauer. Das mediane Gesamtüberleben betrug zum Auswertungszeitpunkt im Median 44,4 Monate. Larotrectinib wirkt auch intrakraniell: So sprachen in der Subgruppe der Patienten mit primären Hirntumoren $36 \%$ auf die Therapie an, in der Subgruppe mit ZNS-Metastasen waren es $60 \%$.
\end{abstract}

Quelle und weitere Infos: www.aerztezeitung.de 